\title{
FidAR-Forum: Eine gute Wahl - mehr Frauen in die Aufsichtsräte
}

\author{
16. September 2009 im ARTrium der Britischen Botschaft in Berlin
}

\begin{abstract}
Agnieszka Goscinska, Projektmitarbeiterin „Erhöhung des Frauenanteils in Führungspositionen deutscher Unternehmen - insbesondere Aufsichtsratspositionen“ des djb, Berlin
\end{abstract}

Eingeladen von der Initiative Frauen in die Aufsichtsräte, FidAR e.V., in Kooperation (u.a.) mit dem Deutschen Juristinnenbund (djb) und gefördert vom Bundesministerium für $\mathrm{Fa}$ milie, Senioren, Frauen und Jugend präsentierten am 16. September 2009 Referentinnen und Referenten aus Wirtschaft, Wissenschaft und Politik sowie der Regierungskommission Deutscher Corporate Governance Kodex (DCGK) Beiträge zur aktuellen Diskussion über die Besetzung des Aufsichtsrats mit weiblichen Mitgliedern.

Inspiriert von der norwegischen Quotenregelung bei der Besetzung des Managements, setzt sich auch FidAR für eine Quote von Frauen für alle Aufsichtsgremien von Aktiengesellschaften und die Aufnahme einer sanktionierbaren Verpflichtung in den DCGK ein. Auch Qualifizierungsangebote und die Stärkung der Kompetenzen aller Aufsichtsratsmitglieder fordert der 2005 gegründete FidAR e.V. „Es hat sich über viele Jahre gezeigt, dass freiwillige Maßnahmen der Wirtschaft nicht ausreichen, um den Frauenanteil in den Führungspositionen der Wirtschaft zu erhöhen “, so FidAR-Präsidentin Monika Schulz-Strelow. „Wir brauchen kurzfristig eine verbindliche Quote von mindestens 25 Prozent Frauen in Aufsichtsräten." Die ausgewogene Vertretung von Frauen in den Aufsichtsräten sei nicht nur ein Gebot der Gleichstellung, sondern vielmehr ein Gebot guter Unternehmensführung.

Das norwegische Modell wurde auf der Veranstaltung von Sven Erik Svedman, Norwegischer Botschafter in Deutschland, vorgestellt: Seit 2006 müssen per Gesetz mindestens 40 Prozent der Sitze im Kontrollgremium der börsennotierten Aktiengesellschaften $(A G)$ von Frauen besetzt sein. In einer Vorlaufphase wurde zunächst auf Freiwilligkeit gesetzt. Ferner wurde zusätzlich eine zweijährige Übergangsfrist für Aktiengesellschaften, die vor dem Inkrafttreten des Gesetzes bereits registriert waren, gewährt, um die erforderliche Quote in den Aufsichtsräten umzusetzen. Börsennotierten Aktiengesellschaften, die diese Quote bis Anfang 2008 nicht erreichen würden, wurden Sanktionen angedroht, die bis zum Verlust der Börsennotierung reichten. Dabei wurde deutlich, dass eine freiwillige Selbstverpflichtung in Norwegen diesen hohen Frauenanteil im Management nicht verwirklichen konnte. „Diese Steigerung hätte ohne das Gesetz wahrscheinlich nicht stattgefunden “, so Svedman. Trotz der erheblichen Widerstände aus der Wirtschaft ,hat Norwegen und auch der überwiegende Teil der norwegischen Wirtschaft mit der Einführung einer gesetzlichen Quote von Frauen in Aufsichtsräten sehr gute Erfahrungen gemacht“, betonte Svedman, auch weil

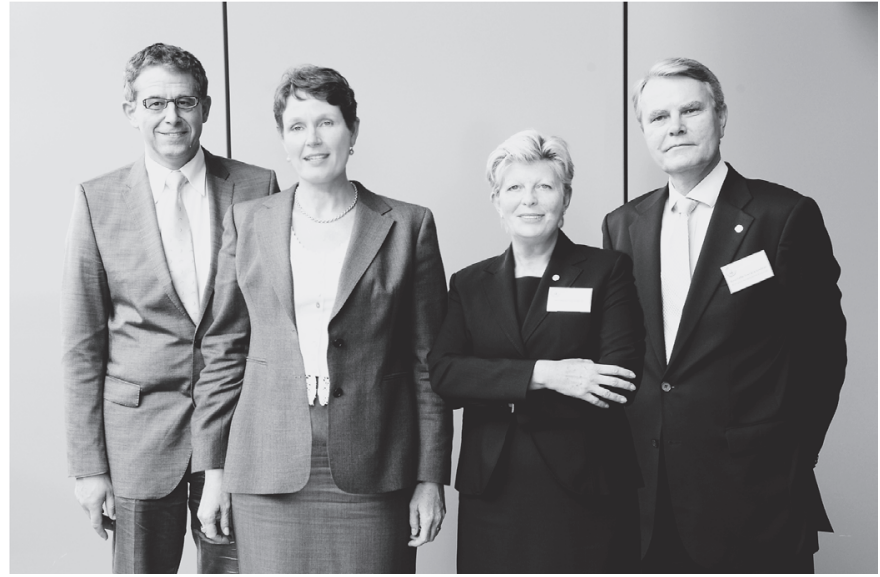

$\Delta$ Begrüßung, Statement und Keynotes von (v.l.n.r.) Gerd Hoofe, Staatssekretär im Bundesministerium für Familie, Senioren, Frauen und Jugend; Daniela Weber-Rey, Mitglied der Regierungskommission Deutscher Corporate Governance Kodex, Monika Schulz-Strelow, Präsidentin von FidAR, Sven Erik Svedman, Norwegischer Botschafter in Deutschland (Foto: Dieter Bühler).

„eine breitere Vielfalt zu größerer Flexibilität, mehr Innovation und zu einer besseren Umsetzungsfähigkeit beiträgt.“

Ebenso hält Eva Maria Welskop-Deffaa, Ministerialdirektorin im Bundesfrauenministerium, die gleichberechtigte Teilhabe von Frauen an Führungspositionen für ein wichtiges Thema. „Es ist skandalös, dass selbst im öffentlichen Dienst, der ja seit Jahren eine Quotenregelung hat, Frauen nur selten die gläserne Decke durchbrechen. Das Thema muss in der nächsten Legislaturperiode auf der politischen Tagesordnung stehen“, so Welskop-Deffaa. Es sei aber wichtig, die Unternehmer mit ins Boot zu holen.

Dr. Carsten Wippermann, Direktor der Sinus Sociovision in Heidelberg, stellte im Rahmen der Veranstaltung erstmals die Ergebnisse der Studie „Brücken und Barrieren für Frauen in Führungspositionen“ im Auftrag des Bundesministeriums für Familie, Senioren, Frauen und Jugend vor. Befragt wurden männliche und weibliche Manager deutscher Unternehmen. Auffällig an der Studie war, dass ein Drittel aller männlichen Führungskräfte gleich mehrfach Karrierestufen übersprungen haben. Bei weiblichen Führungskräften war dieses nicht vergleichbar wiederzufinden.

Eine Unterrepräsentanz von Frauen in Führungspositionen liege nicht an mangelnden Führungsqualitäten von Frauen, so Wippermann. Es liege hauptsächlich an der Mentalität und den Klischees wertkonservativer Männer, die Frauen ausschließen. „Den Männern fallen zwar Gründe gegen Frauen in Aufsichtsräten und Vorstandspositionen ein. Aber keine dafür“, fasst Wippermann zusammen. Die anschließenden Interviews mit den nur männlichen Managern ergaben drei 
Denktypen: Zum einen die mit der ganz schlichten Ansicht, dass Frauen nicht für eine Führungsposition gemacht seien: „Frauen sind ein nicht notwendiges Risiko“ oder „Dieser Konzern duldet keine Frau im Vorstand. Ende. Ist so.“ Dann gebe es noch den Typ, der zwar eine „emanzipierte Grundhaltung " habe, aber trotzdem keine Frau fördern würde aus Angst, dass die Frau aufgrund ihrer Schwäche sich nicht durchsetzen könne. Zuletzt gebe es noch den, der Frauen in Führungspositionen nicht wegen deren Geschlecht ablehne, sondern weil Frauen nicht mehr authentisch blieben. Begründet wurde diese mangelnde Authentizität mit der Logik, dass Frauen in der Unternehmensmännerwelt zur Überkompensation und zu einer unnötigen Strenge neigen würden.

Von einer „interessengeleiteten Nichtwahrnehmung“ sprach Prof. Dr. Stephan Höyng von der Katholischen Hochschule für Sozialwesen Berlin. „Geschlechtsspezifische Diskriminierung nehmen die befragten Männer oft nur selektiv wahr, denn nur so können sie den Widerspruch zwischen ihrer positiven Einstellung zur Gleichstellung und ihrer Untätigkeit bei der Herstellung von Gleichheit aushalten.“

Auch Gerd Hoofe, Staatssekretär im Bundesfamilienministerium, wies auf „Mentalitätsmuster“ hin, „die innerhalb von Unternehmen Hüter der gläsernen Decke sind“. Die Repräsentanz von Frauen in den Führungsetagen müsse transparent gemacht werden. Eine Maßnahme dieser Transparenz ist das Projekt des djb, welches er auf dieser Veranstaltung kurz vorstellte. „Wir können es uns nicht leisten, Chancen zu verschwenden und auf Kompetenzen zu verzichten“, betonte Hoofe. Das Ministerium fördere daher das Projekt des djb, Hauptversammlungen der Aktiengesellschaften zu besuchen und die Vorstände und Aufsichtsräte nach dem Frauenanteil zu fragen. ${ }^{1}$

In die gleiche Richtung zielt bereits heute der DCGK, durch den die Regeln des Aktiengesetzes transparent werden sollen. Die Frankfurter Juristin Daniela Weber-Rey sitzt als einzige Frau in der Regierungskommission. Auf der Veranstaltung sprach sie sich nicht eindeutig für eine Quote aus, sagte jedoch Sätze wie: „Der Kodex ist die Einladung an die Frauen, dies auch einzufordern.“ Auch im Kodex kommt das Wort „Frau“ in dem Absatz nicht vor, in dem es um die Besetzung der Aufsichtsräte geht. Lediglich von „Vielfalt (Diversity)“ ist die Rede. Weber-Rey findet das ausreichend und verwies einerseits auf die Aussagen von Klaus-Peter Müller, dem Vorsitzenden der Regierungskommission DCGK, und andererseits auf die Pressemitteilungen der Kommission. Beide Aussagen beinhalten, dass diese Empfehlung eine größere Internationalität und eine angemessene Vertretung von Frauen erreichen soll. ${ }^{2}$

Prof. Dr. Katrin Hansen verwies auf einige Studien zum Thema Frauen in Führungspositionen. Nach diesen Studien kann „eine starke Minderheitsposition im Aufsichtsrat eine Frau daran hindern, ihre Meinung (effektiv) auszudrücken“. Dem folgend betonte Prof. Dr. Hansen, dass eine Besetzung des Managements paritätisch mit Frauen und Männern erfolgen sollte. „Eine zu geringe Quote führt sonst dazu, dass die Beiträge der Frauen eher missachtet werden.“

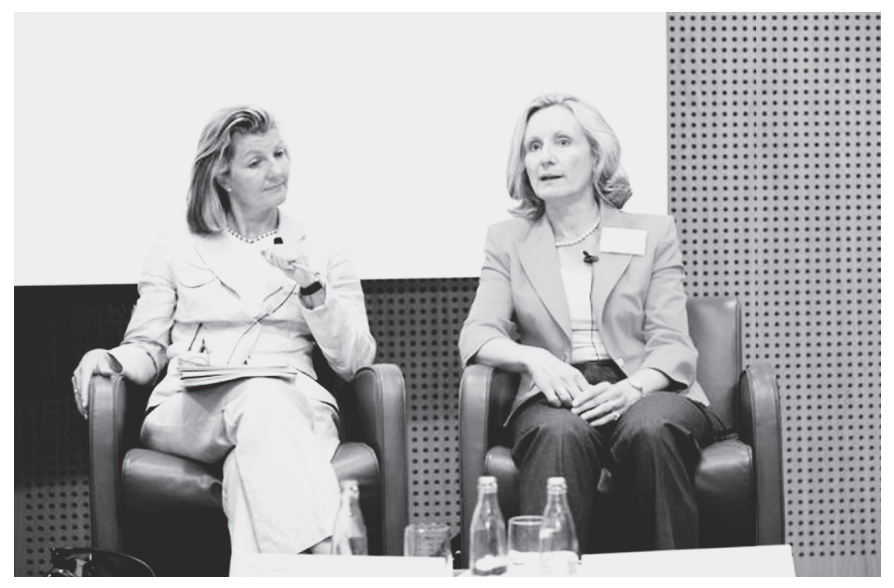

A Auf dem Podium: Jella Benner-Heinacher, Geschäftsführerin Deutsche Schutzvereinigung für Wertpapierbesitz (DSW) in Düsseldorf, Prof. Dr. Marita Körner, Professorin für Wirtschaftsrecht an der Universität der Bundeswehr München und Mitglied im djb (Foto: Dieter Bühler).

Bei der anschließenden Podiumsdiskussion stellte Prof. Dr. Martina Körner, Professorin für Wirtschaftsrecht an der Universität der Bundeswehr München, kurz die aktuelle rechtliche Situation der Geschlechtergleichstellung in Deutschland dar und verwies auf die erfolglosen Selbstverpflichtungen der Unternehmen. Bereits Ende der neunziger Jahre existierten ausformulierte Gesetzentwürfe zur Gleichstellung der Geschlechter in der Privatwirtschaft. Am 8. September 2000 legte die damalige Bundesministerin Dr. Christine Bergmann Eckpunkte für gleichstellungsgesetzliche Regelungen für die Privatwirtschaft vor und kündigte gleichstellungsgesetzliche Regelungen an. ${ }^{3}$ Sie beauftragte eine Expertinnengruppe damit, einen Gesetzentwurf zu erarbeiten, der in den Bundestag eingebracht werden sollte. Die Expertinnengruppe vereinigte mit Prof. Dr. Heide Pfarr, Ingrid Weber, Prof. Dr. Ursula Rust, Prof. Dr. Dagmar Schiek, Dr. Silke Ruth Laskowski und Dr. Eva Kocher djb-Mitglieder unterschiedlicher Praxiserfahrungen und Forschungsschwerpunkte. ${ }^{4}$ Die Bundesregierung entschloss sich jedoch gegen ein Gesetz und für unverbindliche Absichtserklärungen. Der djb beurteilte dieses Vorgehen als „herbe Enttäuschung für alle erwerbstätigen Frauen, die der Koalitionsvereinbarung vertraut und auf gesetzliche Regelungen zur Durchsetzung der Chancengleichheit von Frauen und Männern in der Privatwirtschaft gewartet haben “. 5

1 Ausführlichere Darstellung des Projekts: www.djb.de/Projekte/ Führungspositionen.

2 Nachzulesen auf: www.corporate-governance-code.de.

3 S. „Bundesministerin Bergmann stellt Eckpunkte für Gleichstellungsgesetz in der Wirtschaft vor", Pressemitteilung des BMFSFJ v. 8.9.2000. http://www.bmfsfj.de/bmfsfj/generator/BMFSFJ/Presse/ pressemitteilungen, did=2794.html.

4 S. „Ein Gesetz zur Gleichstellung der Geschlechter in der Privatwirtschaft", hrsg. v. Heide Pfarr, edition der Hans Böckler Stiftung, Düsseldorf 2001, S. 5 ff.

5 Vgl. djb-Pressemitteilung vom 3.7.2001, http://www.djb.de/ Kommissionen/kommission-arbeits-gleichstellungs-undwirtschaftsrecht/pm-29/. 
Für den Beweis des Scheiterns dieses Weges verwies Prof. Dr. Körner auf die „Bilanzen Chancengleichheit“ der Bundesregierung. ${ }^{6}$ Obendrein machte sie auf die Qualitätssteigerung der Aufsichtsräte aufmerksam, „wenn deren Mitglieder aus einem um Kandidatinnen erweiterten Bewerberkreis ausgewählt werden können “. Auch sie ist der Ansicht, dass der „ökonomische Effekt nur eintreten kann, wenn eine signifikante Zahl von Frauen im jeweiligen Führungsorgan vertreten ist. Die Einzelkämpferin reicht nicht".

Damit war das Resümee dieser Veranstaltung, dass es ohne eine gesetzliche Quotenregelung nicht gehen werde, da die deutsche Wirtschaft es sich nicht leisten kann, auf hochkompetente Frauen im Management zu verzichten. Der djb sieht entsprechenden Handlungsbedarf und hält neben gesetzlichen Anforderungen für die Qualifikation von Aufsichtsratsmitgliedern die Einführung einer gesetzlichen Quote von mindestens 40 Prozent für die Besetzung von Aufsichtsratsmandaten mit Frauen für erforderlich.

6 Vgl. die djb-Pressemitteilungen und -Stellungnahmen zu den Bilanzen, zuletzt „Stagnation auf niedrigem Niveau“ v. 26.6.2008, http://www.djb.de/Kommissionen/kommission-arbeitsgleichstellungs-und-wirtschaftsrecht/pmo8_10/.

\title{
Marie Elisabeth Lüders-Preis 2009
}

\author{
verliehen an Dr. Marion Röwekamp \\ am 26. September 2009 in Karlsruhe
}

Im Anschluss an die Mitgliederversammlung des djb am 26. September 2009 begrüßte Präsidentin Jutta Wagner die Gäste im Gartensaal des Karlsruher Schlosses zur Verleihung des „Marie Elisabeth Lüders-Preis 2009“ an Dr. Marion Röwekamp. Sie verlas einen Brief des djb-Gründungsmitglieds Annette Schücking-Homeyer, Richterin i.R. vom September 2009 an den Juristinnenbund:
Der Marie Elisabeth Lüders-Preis wird vom djb in Anerkennung hervorragender rechts- oder wirtschaftswissenschaftlicher Arbeiten alle zwei Jahre vergeben. Er besteht aus einem Druckkostenzuschuss in Höhe von 2.000,- Euro, die in diesem Jahr von Dr. Melitta Büchner-Schöpf gestiftet wurden. Prof. Dr. Sibylle Raasch hielt die Laudatio; Dr. Melitta Büchner-Schöpf übergab die Urkunde an die Preisträgerin. Die beiden Reden und der Dank der Preisträgerin sind im Folgenden abgedruckt.

Dr. Röwekamp erhielt den Preis in Anerkennung ihrer Dissertation „Professionalisierung und Emanzipation. Die kurze Berufsgeschichte der ersten deutschen Juristinnen (1900-1945)“. Sie studierte Geschichte und Jura an den Universitäten Heidelberg, München, der Humboldt Universität zu Berlin und der Columbia Universität, New York. 2005 erschien ihr Buch „Juristinnen. Lexikon zu Leben und Werk“, das vom Deutschen Juristinnenbund herausgegeben wurde. Nach dem Zweiten Juristischen Staatsexamen 2006 in Berlin promovierte sie im Juli 2008 „summa cum laude" mit der jetzt vom djb preisgekrönten Arbeit an der Ludwig-MaximiliansUniversität München. Ihre rechtswissenschaftliche Promotion, eine Biografie über Marie Munk, sozusagen der dritte Band der „Juristinnen-Trilogie“, steht kurz vor dem Abschluss. Seit Anfang September arbeitet sie als John F. Kennedy Fellow am Center for European Studies in Harvard an ihrer Habilitation „Die Ordnung der Familie im Recht 17491933 “. 